\title{
Plasmids, Recombination and Chromosome Mapping in Streptomyces lividans 66
}

\author{
By DAVID A. HOPWOOD, ${ }^{*}$ TOBIAS KIESER, HELEN M. WRIGHT AND \\ MER VYN J. BIBB
}

John Innes Institute, Colney Lane, Norwich NR4 7UH, U.K.

(Received 30 December 1982)

\begin{abstract}
Streptomyces lividans 66 was shown to harbour two self-transmissible plasmids: SLP2, which acts as a sex factor, and SLP3. Derivatives of this strain which had lost both plasmids were used as host strains to study a range of Streptomyces plasmids for their ability to promote their own transfer and to mobilize chromosomal markers. A linkage map of the $S$. lividans chromosome containing ten markers was derived from the results of matings using several different sex plasmids, and protoplast fusions. SLP2 was transferred interspecifically to $S$. parvulus ATCC 12434 and to S. coelicolor A3(2); in the latter it acted as a fertility factor. Interspecific crosses also led to the discovery of a further plasmid, SLP4, from S. coelicolor. SLP2, SLP3 and SLP4 could not be visualized on agarose gels using standard plasmid isolation procedures, but their presence was detected by transformation into $S$. lividans.
\end{abstract}

\section{INTRODUCTION}

Streptomyces lividans 66 has become a standard host strain for gene cloning experiments involving both plasmid and phage vectors (Chater et al., 1982; Hopwood \& Chater, 1982; Bibb et al., 1983). It has several advantages for general cloning purposes over its close relative Streptomyces coelicolor A3(2), which is still genetically the best characterized streptomycete (Hopwood et al., 1973; Chater \& Hopwood, 1983). Notable is the fact that DNA cloning vectors derived from the plasmid SLP1 (Thompson et al., 1982b) cannot be used in S. coelicolor A3(2). This plasmid originates from a chromosomal sequence in $S$. coelicolor A3(2). After mixed culture of $S$. coelicolor with $S$. lividans, SLP1 sequences excised from the chromosome of $S$. coelicolor can be found as autonomous, self-transmissible plasmids in $S$. lividans. Marked SLP1 derivatives can be reintroduced into $S$. coelicolor only at a very low frequency and invariably become integrated into the chromosome (Bibb et al., 1981). A similar phenomenon has recently been discovered when either Streptomyces parvulus ATCC 12434 or Streptomyces glaucescens ETH 22794 is crossed with $S$. lividans 66 , leading to the discovery of the pIJ110 and pIJ408 plasmids (D. A. Hopwood, G. Hintermann, T. Kieser \& H. M. Wright, unpublished). In the course of the present work a further plasmid, SLP4, from S. coelicolor was discovered by mating into $S$. lividans.

Most plasmid or phage DNA grown in Escherichia coli can be introduced into $S$. lividans 66, but not directly into $S$. coelicolor A3(2), presumably because of restriction. However, $S$. coelicolor A3(2) only slightly restricts DNA from $S$. lividans 66 while $S$. lividans is not known to restrict DNA from any other streptomycete. Additional advantages of $S$. lividans 66 are its slightly faster growth rate and sporulation and its performance as a more reliable indicator for the plasmidrelated lethal zygosis $\left(\mathrm{Ltz}^{+}\right)$phenotype than $S$. coelicolor A3(2).

In view of the importance of $S$. lividans 66 as a subject for genetic work, we set out to determine its plasmid content. Two plasmids, SLP2 and SLP3, were found and subsequently eliminated from the strain. The resulting presumably plasmid-free strains and their genetically marked derivatives have been used to develop a preliminary chromosomal linkage map and they 
are presently the preferred hosts in which to study the determination of functions such as stability, compatibility, transfer, lethal zygosis and chromosomal mobilization by particular Streptomyces plasmids with minimal risk that such functions might be provided in trans by unidentified plasmids in the host strain, making interpretation of the experiments difficult.

\section{METHODS}

Bacterial strains, media, culture conditions and genetic techniques. The strains are described in Table 1 and the plasmids in Table 2. Cultural and genetic techniques were those of Hopwood (1967). Auxotrophic mutants were isolated by standard procedures after UV irradiation to $0 \cdot 1-1 \%$ survival. Spontaneous streptomycin- or spectinomycin-resistant mutants were isolated by plating spores on CM (Hopwood, 1967) containing $100 \mu \mathrm{g}$ streptomycin or spectinomycin $\mathrm{ml}^{-1}$. Crosses were made on slants of R2YE medium (Thompson et al., 1980) supplemented with growth factors required by auxotrophic strains: recombinants were recovered on suitably supplemented MM (Hopwood, 1967). (In MM, streptomycin and spectinomycin were used at 4 and $50 \mu \mathrm{g} \mathrm{ml}^{-1}$ respectively.) Protoplasts were prepared from mycelium grown in yeast extract/malt extract medium (YEME) containing $34 \%(\mathrm{w} / \mathrm{v})$ sucrose and $0.5 \%(\mathrm{w} / \mathrm{v})$ glycine (Bibb et al., 1977). Lysozyme treatment was done in $\mathrm{L}$ medium (Thompson et al., 1982a) and the protoplasts were stored in P medium (Okanishi et al., 1974; Hopwood \&

Table 1. Streptomyces strains

\begin{tabular}{|c|c|c|}
\hline Strain (J.I. stock no.) & Chromosomal markers & Plasmids \\
\hline \multicolumn{3}{|l|}{ S. lividans 66} \\
\hline 1326 & - & SLP2, SLP3 \\
\hline TK 19 & - & SLP3 \\
\hline TK20 & - & SLP2 \\
\hline TK21 & - & - \\
\hline TK 24 & str-6 & - \\
\hline TK54 & his-2 leu-2 spc-1 & - \\
\hline TK64 & pro-2 str-6 & - \\
\hline TK65 & cys -8 str-6 & 一 \\
\hline TK68 & ath-8 str-6 & - \\
\hline TK69 & ura-6 str-6 & - \\
\hline TK 70 & $m e t-7$ str-6 & - \\
\hline TK 71 & $i l v-3$ str -6 & - \\
\hline TK219 & his-2 spc-1 & SLP4 \\
\hline 3077 & his-2 leu-2 spc-1 & SLP2 \\
\hline 3078 & his-2 leu-2 spc-1 & pIJ303 \\
\hline 3084 & his-2 leu-2 spc-1 & pIJ6 \\
\hline 3086 & his-2 leu-2 spc-1 & SCP2* \\
\hline 3090 & pro-2 str-6 & SLP2 \\
\hline 3091 & pro-2 str-6 & pIJ303 \\
\hline 3092 & pro-2 str- 6 & plJ6 \\
\hline 3093 & pro-2 str-6 & SCP2* \\
\hline 3104 & pro-2 str-6 & - \\
\hline 3159 & his-2 leu-2 spc-1 & pIJ 110 \\
\hline 3167 & pro-2 str-6 & pIJ 110 \\
\hline 3169 & his-2 leu-2 spc-1 & SLP4 \\
\hline 3173 & pro-2 str-6 & SLP3 \\
\hline 3197 & his-2 leu-2 ura-6 ath-8 str-6 & pIJ303 \\
\hline 3198 & his-2 leu-2 ura-6 ath-8 str-6 & - \\
\hline 3199 & $h i s-2$ leu-2 $s p c-1$ & SCP1 \\
\hline 3200 & pro-2 str-6 & SCP1 \\
\hline 3204 & pro-2 str-6 & pIJ 408 \\
\hline 3206 & his-2 leu-2 spc-1 & pIJ408 \\
\hline M170 & - & SLP1.1, SLP2, SLP3 \\
\hline M180 & - & SLP1.2, SLP2, SLP3 \\
\hline \multicolumn{3}{|l|}{$S$. coelicolor A3(2) } \\
\hline M124 & proAl $\arg A 1$ cys $D 18$ & - \\
\hline M130 & hisAl uraAl strAl & - \\
\hline M171 & hisAl uraAl strAl & SLP2 \\
\hline \multicolumn{3}{|l|}{ S. parvulus ATCC 12434} \\
\hline 2283 & str-I & - \\
\hline M226 & str-1 & SLP2 \\
\hline
\end{tabular}


Table 2. Plasmids

Plasmid

SCP1

SCP2

SCP2*

SLP1.1, etc.

SLP2

SLP3

SLP4

pIJ6

pIJ408

pIJ110

pIJ101

pIJ303
Characteristics

Autonomous plasmid in $S$. coelicolor A3(2)

Autonomous plasmid in $S$. coelicolor A3(2)

Variants of SCP2 with enhanced fertility properties

Family of plasmids from integrated sequence in

$S$. coelicolor $\mathrm{A} 3(2)$ becoming autonomous in

S. lividans 66

Autonomous plasmid in S. lividans 66

Autonomous plasmid in $S$. lividans 66

Plasmid of $S$. coelicolor A3(2) detected after transfer into $S$. lividans 66

SLP1.2 carrying thiostrepton resistance

Plasmid from integrated sequence in $S$. glaucescens

ETH 22794, autonomous in S. lividans 66

Plasmid from integrated sequence in $S$. parvulus

ATCC 12434, autonomous in S. lividans 66

Autonomous plasmid from S. lividans ISP 5434

pIJ101 carrying thiostrepton resistance
Reference

Vivian (1971)

Bibb et al. (1977); Schrempf \&

Goebel (1977)

Bibb et al. (1977)

Bibb et al. (1981)

This paper

This paper

Bibb et al. (1981) and this paper

Thompson et al. (1980)

D. A. Hopwood et al., unpublished

D. A. Hopwood et al., unpublished

Kieser et al. (1982)

Kieser et al. (1982)

Wright, 1978). Protoplast fusion was done by the method of Hopwood \& Wright (1978) using 50\% (w/v) PEG 1000 (without DMSO); samples of the fusion mixture were diluted in P medium and plated directly on R2YE containing required growth factors.

Plasmid isolation and transformation. Plasmid DNA was isolated by a rapid procedure (T. Kieser, unpublished). Mycelium was grown for $16-24 \mathrm{~h}$ at $30^{\circ} \mathrm{C}$ in $50 \mathrm{ml}$ Tryptone Soya Broth (Oxoid CM129), harvested by centrifugation, washed once with $0.3 \mathrm{M}$-sucrose and resuspended in a final volume of $5 \mathrm{ml} 0.3 \mathrm{M}$-sucrose, $0.025 \mathrm{M}$ Tris $/ \mathrm{HCl}(\mathrm{pH} 8), 0.025 \mathrm{~m}-\mathrm{EDTA}(\mathrm{pH} 8)$ and lysozyme $\left(2 \mathrm{mg} \mathrm{ml}^{-1}\right)$. After $1 \mathrm{~h}$ at $37^{\circ} \mathrm{C}, 3 \mathrm{ml} \mathrm{0.3} \mathrm{M}-\mathrm{NaOH}, 2 \%(\mathrm{w} / \mathrm{v})$ sodium dodecyl sulphate was added, followed by immediate mixing by repeated pipetting. The sample was then heated to $80^{\circ} \mathrm{C}$ for $10 \mathrm{~min}$, cooled to room temperature and extracted with $0.8 \mathrm{ml}$ phenol/chloroform $(500 \mathrm{~g}$ Analar phenol, $200 \mathrm{ml}$ distilled water, $500 \mathrm{ml}$ chloroform, $0.5 \mathrm{~g}$ 8-hydroxyquinoline). DNA from the aqueous phase was precipitated for $5 \mathrm{~min}$ at room temperature by addition of $0.7 \mathrm{ml} 3 \mathrm{M}$-sodium acetate (unbuffered) and $7 \mathrm{ml}$ isopropanol, redissolved in $5 \mathrm{ml} \mathrm{0.01} \mathrm{M}$-Tris $/ \mathrm{HCl}+0.001 \mathrm{M}$-EDTA $(\mathrm{pH} 8)$ and reprecipitated for $15 \mathrm{~min}$ at room temperature by adding $0.25 \mathrm{ml} 0.1 \mathrm{M}$-spermine tetrahydrochloride (Hoopes \& McClure, 1981). Spermine was removed from the precipitate by extraction with $10 \mathrm{ml}$ extraction buffer $[70 \mathrm{ml}$ ethanol $+30 \mathrm{ml} 0.3 \mathrm{M}$-sodium acetate ( $\mathrm{pH} 8), 0.01 \mathrm{M}-\mathrm{MgCl}_{2}$ ].

\section{RESULTS}

\section{Detection of two plasmids SLP2 and SLP3 in S. lividans 66}

An early indication that $S$. lividans 66 might harbour an extrachromosomal sex factor came from the observation that the frequency of recombination in crosses between the SCP1- SCP2S. coelicolor A3(2) strains M130 and M124 was increased 300 times when spores of strain 1326 were added to the cross. We then attempted to isolate derivatives of strain 1326 that had lost the sex factor. The assumption was that the parent strain 1326 would elicit lethal zygosis on its plasmid-free derivatives. Since protoplasting and regeneration was known to induce loss of SCP1 and SCP2 from S. coelicolor (Hopwood, 1981a) we regenerated single colonies from protoplasts of strain 1326 (which had been stored at $4{ }^{\circ} \mathrm{C}$ for $7-14 \mathrm{~d}$ for potential enhancement of plasmid loss). Spores of such colonies were then inoculated as patches on plates of R2YE and a drop of spore suspension of strain 1326 was placed in the centre of each patch. After incubation, the cultures were examined for possible zones of lethal zygosis elicited on each patch by strain 1326. Out of 350 patches tested, 18 showed inhibition zones, which were clearly of three types; one type (5 patches) had a narrow white zone of non-sporulating aerial mycelium, visible against the grey sporulating background, about $1.5 \mathrm{~mm}$ from the edge of the 1326 culture (Fig. 1). The second type (5 patches) had a broad white zone of inhibition close to the 1326 culture, while the third ( 8 patches) was represented by a very narrow, sharply-defined zone, intensely red pigmented on the reverse side of the culture (Fig. 2). Subsequent studies showed that the first 


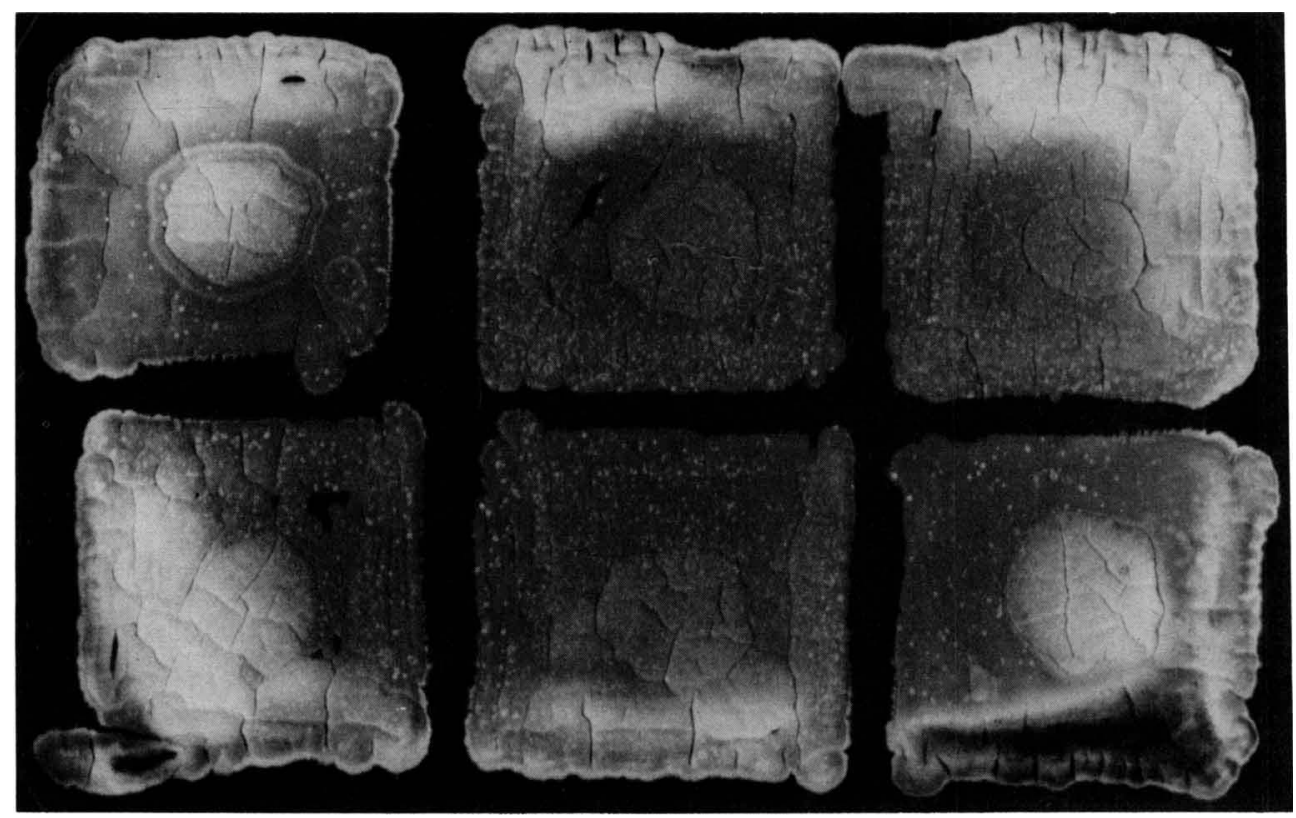

Fig. 1. Patches derived from individual regenerated protoplasts of $S$. lividans strain 1326 inoculated centrally with a drop of spore suspension of strain 1326. Note (top left) a patch of an SLP2- SLP3 ${ }^{+}$ strain showing SLP2-specific lethal zygosis; the patch at bottom right is of an SLP2 ${ }^{+}$SLP3 $^{-}$strain showing SLP3-specific lethal zygosis, barely seen from above.(see Fig. 2 for reverse appearance). The other patches show no lethal zygosis reaction. Magnification $\times 2$.

two types of inhibition zones were due to the same plasmid, designated SLP2; the broad white zone of inhibition in the second type was characteristic of strains that had become chloramphenicol sensitive (Freeman et al., 1977) as well as SLP2-. The narrow pigmented zone was attributed to SLP3. Putative SLP2- cultures such as TK19 (like 1326 itself) elicited lethal zygosis of the narrow type on putative SLP $3^{-}$strains such as TK20, while SLP3- cultures (again like strain 1326) caused the broad type of lethal zygosis on SLP2- strains. By a further round of protoplast regeneration SLP2- SLP3- strains (e.g. TK21) were isolated from SLP2 ${ }^{-}$SLP3 ${ }^{+}$ strain TK 19. They were sensitive to both types of lethal zygosis and elicited neither type (Fig. 2). Zones of lethal zygosis elicited by individual SLP2 ${ }^{+}$spores in a SLP2- background ('pocks') are shown in Fig. 3(a).

That both SLP2 and SLP3 were transmissible in mixed cultures of plasmid ${ }^{+}$and plasmid ${ }^{-}$ strains (as expected since lethal zygosis caused by other plasmids has been absolutely correlated with plasmid transfer: Bibb \& Hopwood, 1981; Kieser et al., 1982) was demonstrated in the crosses SLP2+ SLP3- (TK20) × SLP2- SLP3- str-6 (TK24) or SLP2- SLP3+ (TK19) $\times$ SLP2- SLP3- pro-2 str-6 (TK64) plated on medium containing streptomycin to recover the originally plasmid-free parental chromosomal genotype. Samples of the resulting colonies were replicated to lawns of TK24 (or TK64) on R2YE to determine the proportion of $\mathrm{Ltz}^{+}$colonies. SLP2 ${ }^{+}$showed an apparent transfer frequency of $100 \%$ (102 out of 102 tested). For SLP3 the frequency was about $50 \%$ (26 out of 59$)$.

\section{Transfer of SLP2 to other species}

Crosses were made between $S$. lividans TK20 (SLP2 ${ }^{+}$SLP3 $\left.{ }^{-}\right), 1326$ (SLP2 ${ }^{+}$SLP3 $\left.{ }^{+}\right)$, M1 $^{2} 0$ $\left(\mathrm{SLP} 1.1^{+} \mathrm{SLP} 2^{+} \mathrm{SLP}^{+}\right)$or M180 (SLP1.2+ SLP2 $\left.{ }^{+} \mathrm{SLP} 3^{+}\right)$and streptomycin-resistant strains of $S$. coelicolor (M130) or $S$. parvulus (2283). On plating spores from the crosses on R2YE or appropriately supplemented $\mathrm{R} 2$, both containing streptomycin, in the presence of sufficient spores of the M130 or 2283 parent to produce a confluent lawn of growth, distinct pocks were seen, generally resembling those shown by SLP2 in S. lividans (Fig. $3 b, c$ ). Isolation of cultures 

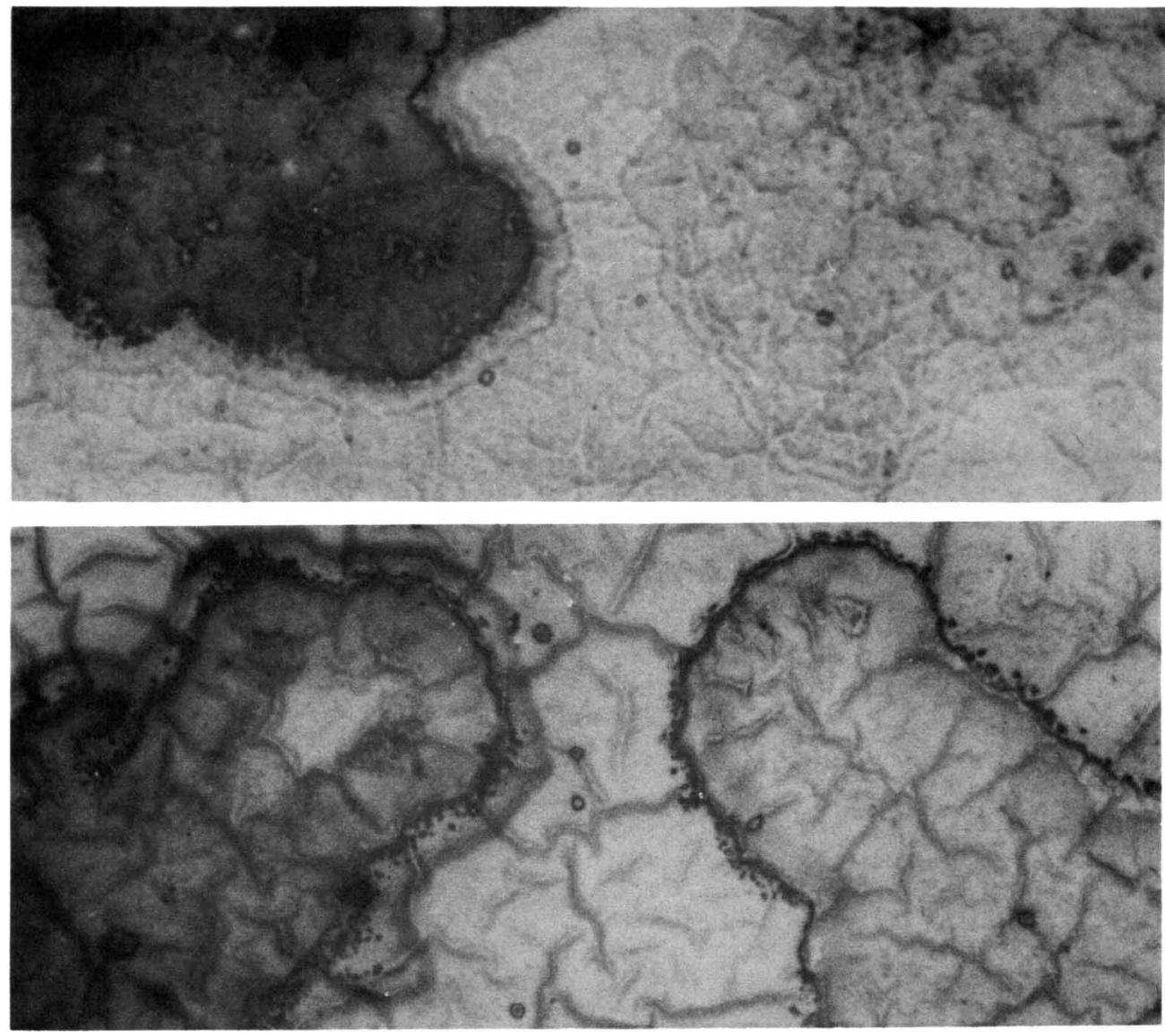

Fig. 2. Replica on SLP2 ${ }^{-}$SLP3 $^{-}$strain TK21 of $S$. lividans of : (top left and bottom left) SLP2 ${ }^{+}$SLP3 $^{+}$ strain 1326; (top right) SLP2+ SLP3- strain TK20; (bottom right) SLP2- SLP3+ strain TK 19. Note SLP2-specific lethal zygosis for patches top left, bottom left and top right, and SLP3-specific lethal zygosis top left, bottom left and bottom right. (Plates viewed from below). Magnification $\times 2$.

from the centres of such pocks gave $S$. coelicolor or $S$. parvulus strains reproducibly eliciting SLP2-type lethal zygosis. Analysis of colonies of $S$. coelicolor and $S$. parvulus resulting from five matings of each with $S$. lividans M180 and M170 respectively for their ability to express lethal zygosis on lawns of the parental type indicated that SLP2 had been transferred interspecifically to $S$. coelicolor and $S$. parvulus at frequencies of $7-38 \%$ and $4-59 \%$ respectively. (The involvement of the SLP1 or SLP3 plasmids in assisting SLP2 transfer in these matings could not be ruled out.) This high frequency of plasmid transfer contrasts with the low level of exchange of chromosomal genes which occurs between strains of $S$. lividans and $S$. coelicolor or $S$. parvulus during mating or protoplast fusion (P. Somasundaram, personal communication). SLP2 was stably maintained in these two species: 0 out of 1136 and 0 out of 950 colonies of an SLP2 ${ }^{+}$ culture of $S$. coelicolor and $S$. parvulus respectively lost the ability to elicit lethal zygosis.

Analysis of interspecific crosses between SLP2 ${ }^{+}$and SLP2- strains of $S$. coelicolor and $S$. parvulus indicated transfer of SLP2 from $S$. coelicolor to $S$. parvulus at similar, high frequencies: $4-93 \%$ of potential recipients had acquired SLP2 in five matings analysed. Transfer of SLP2 from $S$. parvulus to $S$. coelicolor occurred at a much lower frequency, estimated by pock formation to be approximately $10^{-5}$ (one mating analysed), perhaps reflecting the existence of a previously suggested but undefined restriction system in $S$. coelicolor (Hopwood \& Wright, 1973). SLP2+ derivatives of $S$. coelicolor and $S$. parvulus obtained in these latter matings were identical to the original SLP2+ isolates obtained directly by mating with $S$. lividans in pock 

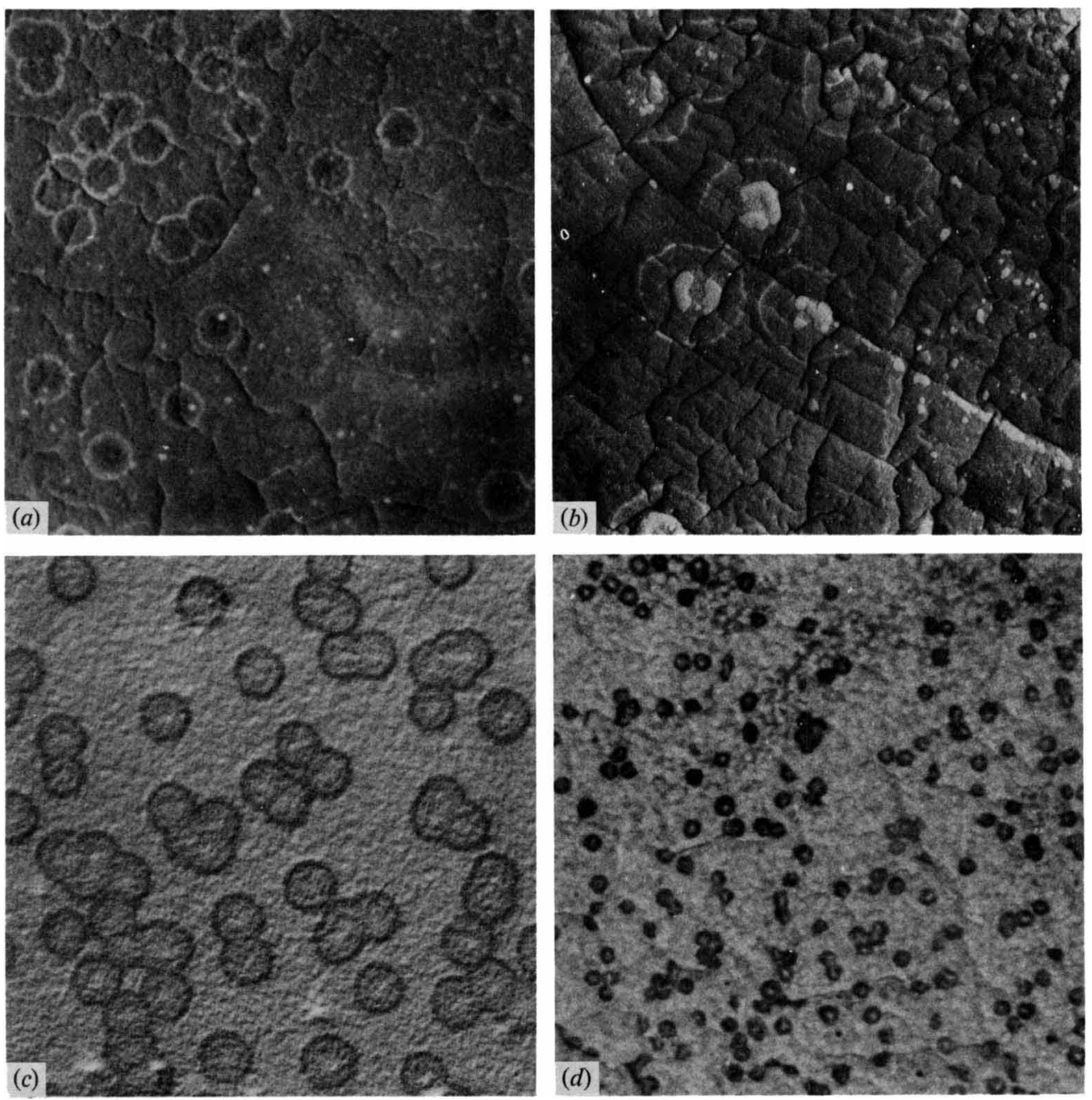

Fig. 3. Lethal zygosis caused by SLP2 in (a) S. lividans, $(b) S$. coelicolor and $(c) S$. parvulus, and $(d)$ by SLP4 in $S$. lividans. $(a),(c)$ and $(d)$ show individual pocks produced by individual plasmid-containing spores germinating in a lawn of plasmid-free spores. (b) shows a replica from plasmid-containing colonies to a lawn of a plasmid-free strain. Magnification $\times 3 \cdot 3$.

morphology and in expression of lethal zygosis against an SLP2- lawn. They were resistant to the lethal zygosis normally produced by the original SLP2 ${ }^{+}$isolates and failed to elicit this effect upon them, indicating that they were true SLP2 recipients.

Reverse transfer of SLP2 from $S$. coelicolor or $S$. parvulus to $S$. lividans was not tested since such experiments would have been compromised by the ability of the SLP1 sequences of $S$. coelicolor (Bibb et al., 1981) and the pIJ110 plasmid of $S$. parvulus (D. A. Hopwood, G. Hintermann, T. Kieser \& H. M. Wright, unpublished) to give rise to pocks upon transfer to $S$. lividans.

\section{A new plasmid, SLP4, in S. coelicolor A3(2)}

Mutations (ltz) in the chromosomally integrated SLP1 sequence of $S$. coelicolor can be isolated which prevent the formation of SLP1 pocks in S. lividans (Bibb et al., 1981). However, such $S$. coelicolor mutants do generate pocks of a different kind in S. lividans: they have a much smaller diameter than those produced by the SLP1 plasmids. These observations led to the recognition of a new plasmid, SLP4, originating in S. coelicolor A3(2) and transferable to S. lividans 66 (Bibb et al., 1981). 
Pocks produced by SLP4 in S. lividans are shown in Fig. $3(d)$. In mating experiments, an apparent transfer frequency of about $60 \%$ (311 out of 525 colonies) was observed.

\section{Transformation by SLP2, SLP3 and SLP4 DNA}

Several attempts to demonstrate extrachromosomal DNA corresponding to SLP2, SLP3 or SLP4 by alkaline lysis of strains TK20 (SLP2 ${ }^{+}$), TK 19 (SLP3 ${ }^{+}$) and TK219 (SLP4 ${ }^{+}$), followed by agarose gel electrophoresis, were unsuccessful. Preparations from each strain obtained by following the plasmid isolation procedure were used to transform protoplasts of strain TK24 (SLP2- SLP3- ${ }^{-}$SLP4 $^{-}$) and the protoplasts were allowed to regenerate into confluent cultures. No unambiguous pocks were seen on these primary regeneration plates. However, on replating diluted spore suspensions from such cultures in the presence of excess TK24 spores, pocks characteristic of SLP2, SLP3 or SLP4, respectively, were seen. In similar experiments, $1 \mu \mathrm{g}$ samples of total DNA prepared from $S$. coelicolor strains M171 (SLP2 ${ }^{+}$) and M130 (SLP2-), $S$. parvulus M226 (SLP2 ${ }^{+}$) and 2283 (SLP2-) and S. lividans $1326\left(\right.$ SLP3 $^{+}$SLP2 ${ }^{+}$) were used to transform protoplasts of $S$. coelicolor M130, S. parvulus 2283 and $S$. lividans 1326. After replating spores from the primary regeneration plates on appropriately supplemented $R 2$ with an excess of parental spores, all of the donor DNA samples predicted to contain SLP2 DNA gave rise to SLP2 pocks characteristic of the species concerned. Samples of total DNA isolated from M171, M226 and 1326 each gave rise to identical SLP2 pocks after transformation of M130 or 2283 protoplasts. These SLP2 transformants were identical in their ability to express, and be resistant to, lethal zygosis to the SLP2 recipients obtained after interspecific mating. DNA prepared from SLP2- strains M130 and 2283 failed to elicit SLP2 pock formation. As expected, since the strain already contained the plasmid, attempted transformation of protoplasts of 1326 (SLP2 ${ }^{+}$) with each of the DNA samples failed to give rise to SLP2-like pocks. These experiments, while not allowing quantification, demonstrated that biologically active DNA corresponding to each plasmid could be extracted from $S$. lividans cultures, even if not in quantities capable of physical detection.

\section{Chromosomal recombination mediated by various plasmids in $S$. lividans}

In crosses of the two plasmid-free (i.e. SLP2- SLP3-) strains, TK54 (his-2 leu-2 spc-1) and TK64 (pro-2 str-6), analysed by plating on four media, each selective for recombinants carrying a marker from each parent, no recombinants were found when a frequency of $4 \times 10^{-9}$ would have been detected (Kieser et al., 1982). Such SLP2- SLP3- $S$. lividans strains were used to measure chromosomal recombination attributable to each of several plasmids. By conjugation of strains TK54 and TK64 with various donors, or in the cases of pIJ6, SCP2*, pIJ303, PIJ408 and pIJ110 by transformation, a series of strains were prepared each carrying a different one of the following plasmids (Table 2): pIJ6 (a derivative of SLP1.2 unaffected in lethal zygosis phenotype); SLP2; SLP3; SLP4; SCP1; SCP2*; pIJ303 (a derivative of pIJ101 unaffected in lethal zygosis phenotype); pIJ110 and pIJ408. Crosses were then made of the type his-2 leu-2 $s p c-1$ plasmid $^{+} \times$pro-2 str-6 plasmid $^{-}$and/or his-2 leu-2 spc-1 plasmid ${ }^{-} \times$pro-2 str-6 plasmid $^{+}$. Progeny were plated on two media selecting for recombinants, between his ${ }^{+}$and $s p c$ and between $\mathrm{leu}^{+}$and $\mathrm{spc}$ (these pairs of markers are far apart on the linkage map: see below), and on two media selecting for the parental genotypes. Recombination frequencies were calculated by dividing the average recombinant count by the sum of the parental counts. The results (Table 3 ) showed that all the plasmids, except SLP3 and SLP4, promoted chromosomal recombination. For SLP1 (represented by pIJ6), SLP2, SCP2*, pIJ408 and pIJ110 the frequencies were around $10^{-4}$ (average $1.8 \times 10^{-4}$ ). For pIJ101 (represented by pIJ303), the frequency was higher, at about $5 \times 10^{-3}$, and for SCP1 it was lower, at $5 \times 10^{-6}$.

For the seven plasmids that promoted recombination, crosses of the type his-2leu-2spc-1 plasmid $^{+} \times$pro- 2 str- 6 plasmid ${ }^{+}$were made for comparison with those in which the plasmid was present in only one parent in the cross. The results (Table 3 ) showed no difference in recombination frequency in plasmid ${ }^{+} \times$plasmid $^{+}$compared with plasmid $^{+} \times$plasmid $^{-}$crosses for SLP1 (pIJ6), SCP1, SCP2*, pIJ101 (pIJ303), pIJ408 and pIJ110; this suggested the absence of 'entry disadvantage' (Kirby, 1976; Bibb \& Hopwood, 1981) for these plasmids. In contrast, 
Table 3. Chromosomal recombination frequencies in crosses between $S$. lividans strains carrying markers his-2 leu-2 spc-1 and pro-2 str-6, caused by various plasmids

Strain nos.
of parents
TK54 $\times$ TK64
TK54 $\times 3173$
$3169 \times$ TK64
$3084 \times$ TK64
TK54 $\times 3092$
$3084 \times 3092$
$3077 \times$ TK64
TK54 $\times 3090$
$3077 \times 3090$
$3199 \times$ TK64
TK54 $\times 3200$
$3199 \times 3200$
$3086 \times$ TK64
TK54 $\times 3093$
$3086 \times 3093$
$3078 \times$ TK64
TK54 $\times 3091$
$3078 \times 3091$
$3159 \times$ TK64
TK54 $\times 3167$
$3159 \times 3167$
$3206 \times$ TK64
TK54 $\times 3204$
$3206 \times 3204$

Plasmid in each parent:

$\begin{array}{cc}\text { his-2 leu-2 spc-1 } & \text { pro-2 str-6 } \\ - & - \\ - & \text { SLP3 } \\ \text { SLP4 } & - \\ \text { pIJ6 } & - \\ - & \text { pIJ6 } \\ \text { pIJ6 } & \text { pIJ6 } \\ \text { SLP2 } & - \\ - & \text { SLP2 } \\ \text { SLP2 } & \text { SLP2 } \\ \text { SCP1 } & - \\ - & \text { SCP1 } \\ \text { SCP1 } & \text { SCP1 } \\ \text { SCP2* } & - \\ - & \text { SCP2* } \\ \text { SCP2* } & \text { SCP2* } \\ \text { pIJ303 } & - \\ - & \text { pIJ303 } \\ \text { pIJ303 } & \text { pIJ303 } \\ \text { pIJ110 } & - \\ - & \text { pIJ110 } \\ \text { pIJ110 } & \text { pIJ110 } \\ \text { pIJ408 } & - \\ - & \text { pIJ408 } \\ \text { pIJ408 } & \text { pIJ408 } \\ & \\ & \end{array}$

Average recombinants $\dagger /$ sum parents

$$
\begin{aligned}
& <4 \times 10^{-9} \ddagger \\
& <2 \times 10^{-9} \\
& <3 \times 10^{-9} \\
& 2.1 \times 10^{-4} \\
& 7.6 \times 10^{-5} \\
& 1.9 \times 10^{-4} \\
& 1.7 \times 10^{-4} \\
& 3.2 \times 10^{-4} \\
& 1.8 \times 10^{-6} \\
& 3.5 \times 10^{-6} \\
& 6.8 \times 10^{-6} \\
& 3.2 \times 10^{-6} \\
& 1.4 \times 10^{-4} \\
& 1.1 \times 10^{-4} \\
& 8.1 \times 10^{-5} \\
& 6.4 \times 10^{-3} \ddagger \\
& 2.1 \times 10^{-3} \ddagger \\
& 4.3 \times 10^{-3} \ddagger \\
& 1.0 \times 10^{-4} \\
& 8.7 \times 10^{-5} \\
& 1.2 \times 10^{-4} \\
& 3.8 \times 10^{-4} \\
& 2.2 \times 10^{-4} \\
& 2.2 \times 10^{-4}
\end{aligned}
$$

$\dagger$ Average on media selecting $h i s^{+} / s p c$ and $l e u^{+} / s p c$.

$\ddagger$ From Kieser et al. (1982).

the frequency of recombination in SLP2 ${ }^{+} \times \mathrm{SLP}^{+}$crosses was 100 times lower than in the corresponding SLP2 ${ }^{+} \times \mathrm{SLP}^{-}$crosses, suggesting an 'entry disadvantage' for SLP2.

The fertility properties of SLP2 in S. coelicolor were also studied. SLP2 was found to promote recombination in $S$. coelicolor at a similar frequency to that found in $S$. lividans. In crosses of SCP1-SCP2- strains M130 and M124, recombinants were found at a frequency of about $5 \times$ $10^{-8}$, as described by Bibb \& Hopwood (1981). When SLP2 was present in one parent (M171), the frequency was increased approximately $10^{3}$-fold to about $5 \times 10^{-5}$. Transfer of SLP2 from donor to recipient occurred almost invariably at a frequency of $100 \%$. Analysis of the progeny of crosses between $S$. coelicolor derivatives containing SLP2 and strains of complementary genotype containing SCP2, SCP2* or SCP1 failed to demonstrate any interaction between SLP2 and any of the other sex factors, expressed either as an effect on plasmid transfer or in the observed levels of chromosomal recombination. Similarly, SLP2 did not exhibit detectable incompatibility with any of the other three plasmids.

\section{A linkage map for $S$. lividans 66}

The simplest reliable method of developing a preliminary linkage map of a new streptomycete is to analyse matings between pairs of strains, each carrying two selectable alleles, by plating on the four media each selective for one marker from each parent, with two other markers nonselected on each medium (the 'four-on-four' procedure: Hopwood, 1959). Classification of samples of the recombinants arising on each medium into the four possible genotypes in respect of non-selected markers normally allows an unambiguous circular linkage map in respect of the four markers to be drawn, with some indication of their relative spacing (Hopwood, 1972). This procedure was successfully applied to $S$. lividans and it was found that any one of the seven fertility plasmids, pIJ6, SLP2, SCP1, SCP2*, pIJ303, pIJ408 or pIJ110, could be used to 'drive' the crosses. An example of a cross involving markers his-2 leu-2 spc-1 and pro-2, with SLP2 as 
Table 4. Analysis of recombination in cross 3077 (his-2 leu-2 spc-1 SLP2 $\left.2^{+}\right) \times$ TK64 (pro-2 str-6)

For the method of analysis, see Hopwood (1972).

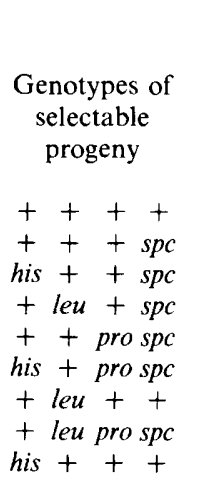

Sample size:

Total recombinants per plate:
Selective media supplemented with:

\begin{tabular}{|c|c|c|c|c|c|}
\hline \multicolumn{2}{|c|}{ Histidine } & \multicolumn{2}{|c|}{$\begin{array}{c}\text { Leucine, } \\
\text { proline, } \\
\text { spectinomycin }\end{array}$} & \multicolumn{2}{|c|}{ Leucine } \\
\hline$a$ & $b$ & $a$ & $b$ & $a$ & $b$ \\
\hline 6 & 4 & - & - & 2 & 6 \\
\hline 58 & 35 & 13 & 80 & 13 & 36 \\
\hline 36 & 22 & - & - & - & - \\
\hline- & - & 28 & 171 & 75 & 206 \\
\hline- & - & 59 & 361 & - & - \\
\hline- & - & - & - & - & - \\
\hline - & - & - & - & 10 & 27 \\
\hline- & - & 0 & 0 & - & - \\
\hline 0 & 0 & - & - & - & - \\
\hline
\end{tabular}

100

spect

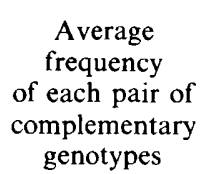

proline pectinomycin

$a \quad b$

$5 \quad 27$

316

$\begin{array}{ll}- & - \\ 67 & 367\end{array}$

$\left.\begin{array}{ll}25 & 137\end{array}\right\}$

$\left.\begin{array}{cc}25 & 137 \\ - & - \\ - & - \\ - & -\end{array}\right\}$

100

$61 \quad 612 \quad 275$

547

Segregation of pairs of non-selected alleles

Arrangement deduced:

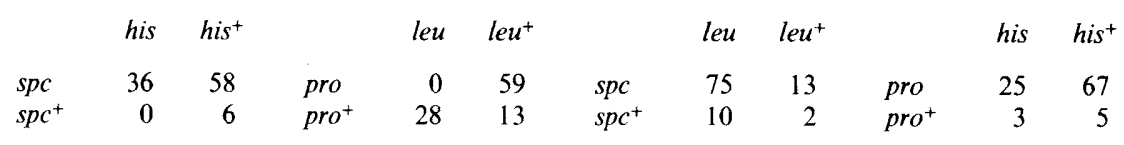

Adjacent

Adjacent

Not adjacent

Not adjacent

Relative recombination frequency in each interval

$\begin{array}{cccccc}\text { pro-leu } & \text { leu-his } & \text { pro-his } & \text { pro-spc } & \text { leu-spc } & \text { his-spc } \\ 69 & 290 & 321 & 451 & 510 & 598\end{array}$

the sex factor, is given in Table 4, leading to the arrangement of the four markers as shown in Fig. 4. In spite of the fact that crosses involving any one of the sex plasmids yielded the same circular sequence of these four markers, the apparent recombination in different intervals varied considerably; however, a much more extensive study would be needed to discriminate effects of the distribution of crossovers between various map intervals, selective effects, etc.

Five more markers were added to the linkage map by analysing further four-factor crosses of strain 3077 (his-2 leu-2 spc-1 SCP2 ${ }^{+}$) with each of the strains TK65 (cys-8), TK68 (ath-8), TK69

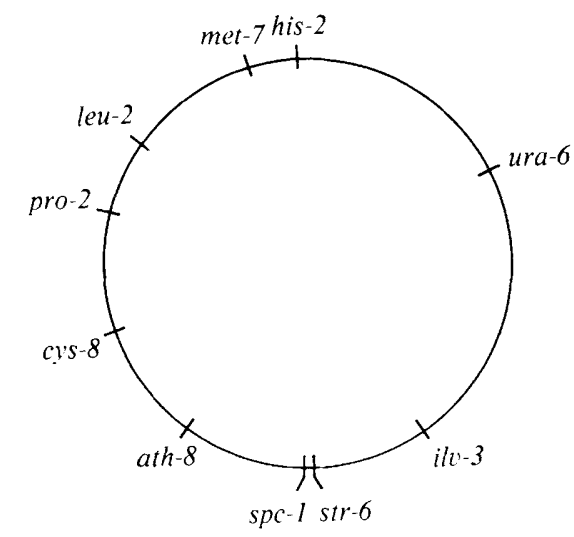

Fig. 4. Linkage map of $S$. lividans for ten markers. Map distances have not been determined precisely. 
(ura-6), TK70 (met-7) and TK71 (ilv-3). met-7 fell between leu-2 and his-2 and closer to the latter; ura- 6 and $i l v-3$ both fell between his- 2 and $s p c-1$, with ile-3 quite close to $s p c-1$ and ura- 6 closer to his-2; cys- 8 and ath- 8 both fell between $s p c-1$ and $l e u-2$, with ath- 8 much closer to $s p c-1$ and $c y s-8$ closer to leu-2. In each cross, str-6 was a supernumerary non-selected marker, which turned out to be close to $s p c-1$ ( 33 out of $1464=2 \cdot 3 \%$ recombinants). The linkage relations of the ten markers are summarized in Fig. 4.

\section{Comparison of plasmid-mediated conjugation and protoplast fusion for linkage mapping}

Strains carrying five or six markers were constructed by two rounds of protoplast fusion between SLP2- SLP3- strains: TK54 (his-2 leu-2 spc-1) was fused with TK69 (ura-6 str-6) to yield 3102 (his-2 leu-2 ura-6 spc-1) and TK68 (ath-8 str-6) was fused with TK71 (ilv-3 str-6) to yield 3103 (ath-8 ilv-3 str-6). Fusion of 3102 with 3103 yielded 3106 (his-2 leu-2 ura-6 ath-8 ilv-3 str-6) and 3198 (his-2 leu-2 ura-6ath-8 str-6). Matings of either of these strains with strains carrying new mutations (with a suitable sex plasmid in one or both parents) were found to be suitable for the mapping of such mutations; however, the ilv-3 marker conferred a selective disadvantage on some genotypes of recombinant progeny and so strain 3198 was preferred for most crosses.

Table 5 and Fig. 5 show a comparison of the results of a seven-factor protoplast fusion between SLP2- SLP3- strains 3198 and 3104 with those of a mating between the corresponding strains 3197 (equivalent to 3198 but carrying pIJ303 as a sex plasmid) and 3104. In each case, recombinants inheriting his- $2^{+}$and $s t r-6$ were selected. In Table 5, the $s p c-1$ marker (close to str- 6 ) has been ignored. We see that, in the mating, only 2 out of the 286 progeny $(0.7 \%)$ arose by multiple (quadruple) crossing-over, whereas in the protoplast fusion, 46 out of $344(13 \%)$ were multiples. [In a six-factor protoplast fusion in S. coelicolor, analysed non-selectively, Hopwood \& Wright (1978) found $24 \%$ multiple crossover genotypes amongst the recombinants; the two results are in good agreement since in the $S$. lividans example studied here only half the number of multiple crossover genotypes were detectable because three non-selected markers were in one arc between the selected markers and only one was in the other arc.] The higher frequency of crossing-over in the protoplast fusion compared with the mating is also reflected in the allele ratios in Fig. 5(b), which are much closer to $50: 50$, even for loci close to the points of selection, than in Fig. 5(a).

The more random distribution of crossovers over the various map intervals in protoplast fusions compared with matings (Hopwood \& Wright, 1978) is apparent in Table 5. For example, in the mating, there is a significant tendency for crossovers in interval 4 to be associated with crossovers in the adjacent interval 5, compared to the non-adjacent interval 6 (16 compared with 0 ); in the protoplast fusion, this tendency was much less marked (62 compared with 27). This result presumably refiects the incomplete nature of the merozygotes produced by conjugation compared with the complete diploids arising by protoplast fusion (Hopwood \& Wright, 1978; Hopwood, $1981 b$ ).

The suitability of either mating or protoplast fusion for the location of a new marker on the linkage map can be illustrated by assuming that one marker (say pro-2) is to be mapped on a linkage group already containing the other five genes. Comparing the allele frequency for pro-2 with that for the other markers we see (Fig. $5 a, b$ ) that pro-2 must be rather close to either $l e u^{+}$or $\mathrm{ura}^{+}$. Analysis of the segregation of $\mathrm{pro}^{+} / \mathrm{pro}$ with that of either leu/leu+ or ura/ura+ (Fig. 5) shows near-independent segregation at the pro and ura loci but a very significant departure from independence for pro and leu, with a marked deficiency of the leu pro genotype. The conclusion is that pro-2 lies close to, and anticlockwise of, leu-2 on the linkage map; and this emerges unambiguously from the results of both the mating and the protoplast fusion (so clearly that a progeny of only a few tens of recombinants would have been enough to establish this result).

The progeny were also classified in respect of the $s p c-1$ marker, which was already known to be very close to $s t r-6$; an analysis of the segregation of $s p c$ in relation to markers on either side of the spc-str region, ath and ura, is at the bottom of Fig. 5. We see that only 9 recombinant progeny $(3 \%)$ inherited $s p c$ in the mating (Fig. $5 a$ ), whereas $87(24 \%)$ did so in the protoplast fusion (Fig. $5 b$ ). As a result, there are enough $s p c$ progeny from the protoplast fusion to show essentially 
Table 5. Results of a mating between strains 3197 (his-2 leu-2 ura-6 ath-8 str-6 pIJ303) and 3104 (pro-2 spc-1) and of a protoplast fusion between strains 3198 (his-2 leu-2 ura-6 ath-8 str-6) and 3104 (pro-2 spc-1)

See Fig. 5 for marker arrangement and map intervals. Recombinants inheriting his-2+ and str-6 (indicated by triangles) were selected.

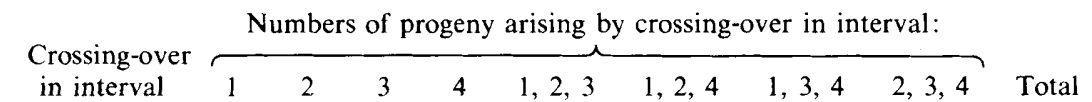

(a) Mating

$\begin{array}{rrrrrrrrrr}5 & 29 & 18 & 123 & 16 & 1 & 0 & 0 & 0 & 187 \\ 6 & 26 & 11 & 61 & 0 & 1 & 0 & 0 & 0 & 99 \\ \text { Total: } & 55 & 29 & 184 & 16 & 2 & 0 & 0 & 0 & 286\end{array}$

(b) Protoplast fusion

$\begin{array}{rrrrrrrrrr}5 & 48 & 29 & 36 & 62 & 2 & 1 & 10 & 8 & 196 \\ 6 & 52 & 13 & 31 & 27 & 8 & 2 & 11 & 4 & 148 \\ \text { Total : } & 100 & 42 & 67 & 89 & 10 & 3 & 21 & 12 & 344\end{array}$
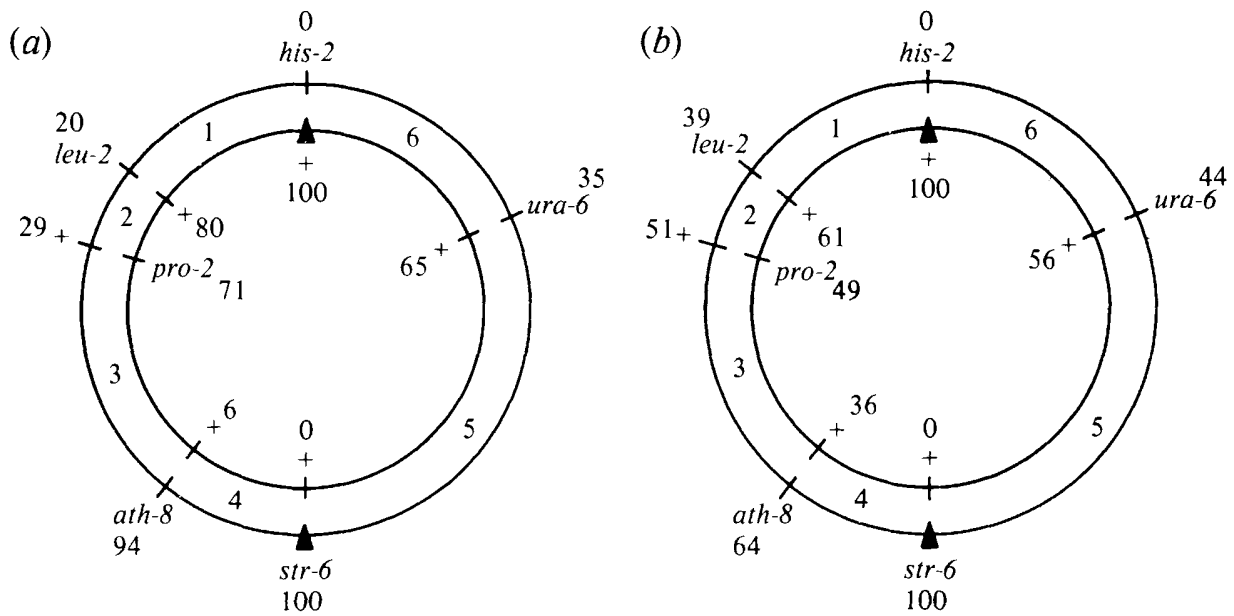

\begin{tabular}{|c|c|c|c|c|c|c|c|c|c|c|c|}
\hline & leu & leu ${ }^{+}$ & & ura & $\mathrm{ura}^{+}$ & & leu & $\mathrm{leu}^{+}$ & & ura & ura ${ }^{+}$ \\
\hline pro $^{+}$ & 55 & 29 & pro $^{+}$ & 37 & 47 & $\mathrm{pro}^{+}$ & 121 & 54 & pro $^{+}$ & 84 & 91 \\
\hline \multirow[t]{2}{*}{ pro } & 2 & 200 & pro & 62 & 140 & pro & 13 & 156 & pro & 68 & 101 \\
\hline & ath & $a t h^{+}$ & & ura & $\mathrm{ura}^{+}$ & & ath & $a t h^{+}$ & & ura & $\mathrm{ura}^{+}$ \\
\hline$s p c$ & 1 & 8 & $s p c$ & 0 & 9 & $s p c$ & 20 & 67 & $s p c$ & 46 & 41 \\
\hline$s p c^{+}$ & 269 & 8 & $s p c^{+}$ & 99 & 178 & $s p c^{+}$ & 199 & 58 & $s p c^{+}$ & 106 & 151 \\
\hline
\end{tabular}

Fig. 5. Analysis of the data in Table 5: (a) mating; (b) protoplast fusion. The numbers next to the markers on the diagrams are percentage frequencies of alleles; the numbers between the circles designate map intervals. The triangles indicate selected alleles. The numbers tabulated below the diagrams are the numbers of recombinants with the indicated combinations of alleles.

independent segregation of $s p c / s p c^{+}$with $u r a / u r a^{+}$, and a clear lack of independence for $s p c / s p c^{+}$ with $a t h / a t h^{+}$, and therefore a position for $s p c-1$ clockwise of str-6. In the mating, while the lack of independent segregation of $s p c / s p c^{+}$and $a t h / a t h^{+}$is quite clear (even though it relies on only 9 progeny), independence or otherwise of the segregation of $s p c / s p c^{+}$and $u r a / u r a^{+}$is not established because of lack of the genotype ura spc in the small group of critical progeny which inherited $s p c$. 


\section{DISCUSSION}

These studies led to the identification, and subsequent elimination, of two plasmids of the 'wild-type' strain $S$. lividans 66 . SLP2 is a self-transmissible plasmid which promotes both chromosomal recombination (this paper) and the transfer of non-transmissible derivatives of other plasmids (Kieser et al., 1982). SLP3 (like the SLP4 plasmid of $S$. coelicolor A3(2)), while being self-transmissible, does not mobilize chromosomal markers at a detectable frequency. (SLP3 had previously been found not to mobilize non-transmissible plasmids: Kieser et al., 1982). SLP2 and SLP3 may be the only plasmids of $S$. lividans 66 , although this cannot be proved. However, the important finding is that SLP2-SLP3- strains are 'sterile' and show no properties associated with any known plasmids. This makes such strains very suitable for the study of the biology of Streptomyces plasmids; use of such strains would minimize the chance that an unrecognized plasmid could interfere with the study of plasmid-determined phenotypes, for example by exerting an incompatibility reaction or by supplying a replication or transfer function 'in trans'. In this context, as in that of gene cloning, it is fortunate that $S$. lividans 66, unlike $S$. coelicolor A3(2), shows little or no evidence of restriction of foreign DNA (Chater et al., 1982), thereby removing a possible barrier to the introduction of a variety of plasmids into derivatives of strain 66 .

The development of the $S$. lividans linkage map, described here, illustrates the relative roles of protoplast fusion and plasmid-mediated mating in linkage mapping (Baltz, 1980). Conjugal recombination, mediated by any one of a variety of sex plasmids, is an efficient way of constructing a preliminary linkage map bearing a series of reference markers, and subsequently mapping new markers to one of the intervals so defined. Protoplast fusion is less convenient for general mapping but, as illustrated here in the ordering of $s t r-6$ and $s p c-1$, the higher frequency of crossing-over following protoplast fusion is advantageous in short-range mapping, an operation rendered difficult in matings by the comparatively low frequency of crossing-over per unit map length in Streptomyces (Hopwood, 1966). Protoplast fusion is also suited to the synthesis of the multiply marked strains required for mapping by mating, since it allows auxotrophic alleles to be combined together under non-selective conditions (Hopwood et al., 1977; this paper). Derivatives of pIJ101 (such as the thiostrepton-marked pIJ303 used here) are particularly suited to mapping studies because of their broad host-range (Kieser et al., 1982) and the very high proportion of recombinants which arise in matings involving these plasmids.

It is interesting (if not unexpected) that the series of 10 markers on the $S$. lividans linkage map (Fig. 4) has an exact counterpart in $S$. coelicolor A3(2): his $A, u r a B$, ilvA, strA, spcA, athA, cys $A$, pro $A$, leu $A$, met $A$. This provides a further example of the conservation of linkage relationships in streptomycetes first noted in a comparison of the maps of $S$. coelicolor and $S$. rimosus (Friend \& Hopwood, 1971).

The methods used here to detect fertility factors in S. lividans by either three-parent or interspecific crosses and to isolate plasmid-free strains by screening for Ltz-sensitive mutants may prove useful for other Streptomyces species, especially since there is no need to introduce genetic markers into the strain under investigation. In a very limited number of studies pock formation following interspecific mating has served to reveal the presence of at least six different plasmids, including SLP1 (Bibb et al., 1981), SLP2 and SLP3, (this paper), SLP4 (Bibb et al., 1981 ; this paper), pIJ110 and pIJ408 (D. A. Hopwood, G. Hintermann, T. Kieser \& H. M. Wright, unpublished). This methodology should assist in the recognition of many other Streptomyces plasmids for which there is no known phenotype and which may be refractory to physical isolation.

We thank Derek Lydiate for helpful comments on the manuscript. T.K. gratefully acknowledges a post-doctoral stipend from the Swiss National Foundation. 


\section{REFERENCES}

BALTZ, R. H. (1980). Genetic recombination by protoplast fusion in Streptomyces. Developments in Industrial Microbiology 21, 43-54.

BıB,, M. J. \& Hopwood, D. A. (1981). Genetic studies of the fertility plasmid SCP2 and its SCP2* variants in Streptomyces coelicolor A3(2). Journal of General Microbiology 126, 427-442.

BibB, M. J., Freeman, R. F. \& Hopwood, D. A. (1977). Physical and genetical characterisation of a second sex factor, SCP2, for Streptomyces coelicolor A3(2). Molecular and General Genetics 154, 155-166.

Bibb, M. J., WARD, J. M., Kieser, T., Cohen, S. N. \& HoPwOOD, D. A. (1981). Excision of chromosomal DNA sequences from Streptomyces coelicolor forms a novel family of plasmids detectable in Streptomyces lividans. Molecular and General Genetics 184, 230 240.

Bibb, M. J., Chater, K. F. \& Hopwood, D. A. (1983). Developments in Streptomyces cloning. In Experimental Manipulation of Gene Expression. Edited by M. Inouyé. New York: Academic Press (in the Press).

Chater, K. F. \& Hopwood, D. A. (1983). Streptomyces genetics. In Biology of the Actinomycetes. Edited by M. Goodfellow, M. Mordarski \& S. T. Williams. London: Academic Press (in the Press).

Chater, K. F., Hopwood, D. A., Kieser, T. \& Thompson, C. J. (1982). Gene cloning in Streptomyces. Current Topics in Microbiology and Immunology 96, 69-95.

Freeman, R. F., BibB, M. J. \& Hopwood, D. A. (1977). Chloramphenicol acetyltransferase-independent chloramphenicol resistance in Streptomyces coelicolor A3(2). Journal of General Microbiology 98, 453465.

FrIEND, E. J. \& Hopwood, D. A. (1971). The linkage map of Streptomyces rimosus. Journal of General Microbiology 68, 187-197.

Hoopes, B. C. \& MCClure, W. R. (1981). Studies on the selectivity of DNA precipitation by spermine. Nucleic Acids Research 9, 5493-5504.

Hopwood, D. A. (1959). Linkage and the mechanism of recombination in Streptomyces coelicolor. Annals of the New York Academy of Sciences 81, 887-898.

HopwOoD, D. A. (1966). Lack of constant genome ends in Streptomyces coelicolor. Genetics 54, 1177-1184.

Hopwood, D. A. (1967). Genetic analysis and genome structure in Streptomyces coelicolor. Bacteriological Reviews 31, 373-403.

Hopwood, D. A. (1972). Genetic analysis in microorganisms. Methods in Microbiology 7B, 29-158.

HOPWOOD, D. A. $(1981 a)$. Uses of protoplasts in the genetic manipulation of streptomycetes. Zentralblatt für Bakteriologie Suppl. 11, 523-531.
HopwOoD, D. A. $(1981 b)$. Genetic studies with bacterial protoplasts. Annual Review of Microbiology 35, 237-272.

Hopwood, D. A. \& Chater, K. F. (1982). Cloning in Streptomyces: systems and strategies. In Genetic Engineering, vol. 4, pp. 119-145. Edited by J. K. Setlow \& A. Hollaender. New York: Plenum.

Hopwood, D. A. \& Wright, H. M. (1973). A plasmid of Streptomyces coelicolor carrying a chromosomal locus and its inter-specific transfer. Journal of General Microbiology 79, 331-342.

HopwoOD, D. A. \& WRIGHT, H. M. (1978). Bacterial protoplast fusion: recombination in fused protoplasts of Streptomyces coelicolor. Molecular and General Genetics 162, 307-317.

Hopwood, D. A., Chater, K. F., Dowding, J. E. \& VIVIAN, A. (1973). Advances in Streptomyces coelicolor genetics. Bacteriological Reviews 37, 371-405.

HopwoOd, D. A., WRight, H. M., BibB, M. J. \& COHEN, S. N. (1977). Genetic recombination through protoplast fusion in Streptomyces. Nature, London 268, 171 174.

Kieser, T., HopwoOd, D. A., Wright, H. M. \& THOMPSON, C. J. (1982). pIJ101, a multicopy broad host-range Streptomyces plasmid: functional analysis and development of DNA cloning vectors. Molecular and General Genetics 185, 223-238.

KIRBY, R. (1976). Genetic studies on Streptomyces coelicolor plasmid one. Ph.D. thesis, University of East Anglia, Norwich, U.K.

OKanishi, M., Suzuki, K. \& Umezawa, H. (1974). Formation and reversion of streptomycete protoplasts: cultural conditions and morphological study. Journal of General Microbiology 80, 389 400.

SCHREMPF, H. \& GOEBEL, W. (1977). Characterisation of a plasmid from Streptomyces coelicolor A3(2). Journal of Bacteriology 131, 251-258.

Thompson, C. J., WARD, J. M. \& Hopwood, D. A. (1980). DNA cloning in Streptomyces: resistance genes from antibiotic producing species. Nature, London 286, 527-529.

THOMPSON, C. J., WARD, J. M. \& HoPWOOD, D. A (1982a). Cloning of antibiotic resistance and nutritional genes in streptomycetes. Journal of Bacteriology 151, 668-677.

Thompson, C. J., Kieser, T., WARD, J. M. \& HoPwOOD, D. A. $(1982 b)$. Physical analysis of antibiotic resistance genes from Streptomyces and their use in vector construction. Gene 20, 51-62.

VIVIAN, A. (1971). Genetic control of fertility in Streptomyces coelicolor A3(2): plasmid involvement in the interconversion of UF and IF strains. Journal of General Microbiology 69, 353-364. 